\title{
CRESCIMENTO INICIAL E NUTRIÇÃO DE CEDRO (Cedrela fissilis VELL.) EM FUNÇÃO DE DOSES DE NITROGÊNIO'
}

\author{
Mariângela Brito Freiberger ${ }^{2}$, Iraê Amaral Guerrini ${ }^{3}$, Gabriela Galetti ${ }^{4}$, Dirceu Maximino Fernandes ${ }^{3}$ e \\ Juliano Corulli Corrêa ${ }^{5}$
}

\begin{abstract}
RESUMO - Objetivou-se avaliar os efeitos de doses de nitrogênio no crescimento inicial, índice SPAD, teor e acúmulo de nutrientes em mudas de cedro. O experimento foi realizado em estufa plástica e as mudas, cultivadas em vasos plásticos preenchidos com $20 \mathrm{dm}^{-3}$ de Latossolo Vermelho, arranjados em blocos casualizados com quatro repetições. Testaram-se as doses de 0,40,80, 120 e $160 \mathrm{mg} \mathrm{dm}^{-3} \mathrm{de} \mathrm{N}$. A adubação até a dose de $160 \mathrm{mg} \mathrm{dm}^{-3} \mathrm{de} \mathrm{N}$ promoveu incremento do índice SPAD e do crescimento inicial das plantas, além de maior absorção de N, P, K, Ca, Mg, S, B, Cu, Fe, Mn e Zn. De modo geral, as menores doses de N limitaram o crescimento, mas não a absorção de nutrientes pelas plantas de cedro. Na folha, o único nutriente afetado foi o B, que teve seu teor reduzido com o aumento das doses de N. O aumento da adubação nitrogenada alterou a distribuição do $\mathrm{Cu}$ nas plantas, uma vez que promoveu aumento do teor de $\mathrm{Cu}$ na raiz e redução de seu teor no caule. Maiores doses de $\mathrm{N}$ promoveram maior acúmulo de todos os nutrientes em função do incremento da produção de matéria seca. A adubação nitrogenada modificou a dinâmica de absorção dos nutrientes em cedro, e, com a dose de $160 \mathrm{mg} \mathrm{dm}^{-3} \mathrm{deN}$, a quantidade absorvida obedeceu à seguinte ordem: $\mathrm{N}>\mathrm{S}>\mathrm{Ca}>\mathrm{K}>\mathrm{Mg}>\mathrm{P}>\mathrm{Fe}>$ $\mathrm{Mn}>\mathrm{B}>\mathrm{Zn}>\mathrm{Cu}$.
\end{abstract}

Palavras-chave: Teor e acúmulo; Macronutrientes e micronutrientes; Índice SPAD.

\section{EARLY GROWTH AND NUTRITION OF CEDAR (Cedrela fissilis VELL.) AS AFFECTED BY NITROGEN RATES}

\begin{abstract}
We aimed to evaluate the effects of nitrogen rates in early growth, SPAD index, content and accumulation of nutrients in cedar seedlings. The experiment was carried out in a plastic greenhouse and the seedlings were grown in plastic pots filled with $20 \mathrm{dm}^{-3}$ of Rhodic Hapludox, arranged inrandomized blocks with four replications. Rates of $0,40,80,120$ and $160 \mathrm{mg} \mathrm{dm}^{-3} \mathrm{~N}$ were tested. Fertilization up to $160 \mathrm{mg} \mathrm{dm}^{-3} \mathrm{~N}$ promoted increases in SPAD index and early growth of plants, beyond greater absorption of $N, P, K, C a, M g, S, B, C u, F e, M n$ and $\mathrm{Zn}$. Overall, lower $N$ rates limited growth but not the absorption of nutrients by cedar plants. In leaves, the only nutrien taffected was the B which their content was reduced with increasing of $N$ rate. Increased of nitrogen fertilization changed the Cu distributionin plants, since increased $C u$ content in roots and reduced their content in stem. Higher $N$ rates promoted higher accumulation of all nutrients due to the increase of dry matter. Nitrogen fertilization changed the dynamic of nutrient absorption in cedar. With $160 \mathrm{mg} \mathrm{dm}^{-3} \mathrm{~N}$, the amount absorbed followed the order: $N>S>C a>K>M g>P>F e>M n>B>$ $\mathrm{Zn}>\mathrm{Cu}$.
\end{abstract}

Keywords: Content and accumulation; Macronutrient and micronutrient; SPAD index.

\footnotetext{
${ }^{1}$ Recebido em 18.11.2011 aceito para publicação em 24.05.2013.

${ }^{2}$ Programa de Pós-Graduação em Agronomia (Agricultura), Universidade Estadual Paulista Júlio de Mesquita Filho, Faculdade de Ciências Agronômicas de Botucatu, FCA/UNESP, Brasil. E-mail: <mariangelabf@ fca.unesp.br>.

${ }^{3}$ Universidade Estadual Paulista Júlio de Mesquita Filho, Faculdade de Ciências Agronômicas de Botucatu, FCA/UNESP, Departamento de Ciência do Solo, Brasil. E-mail: <iguerrini@fca.unesp.br>e < dmfernandes@ fca.unesp.br>.

${ }^{4}$ Programa de Pós-Graduação em Sustentabilidade na Gestão Ambiental, Universidade Federal de São Carlos, UFSCAR, Brasil. E-mail:<gabi.18florestal@gmail.com>.

${ }^{5}$ Empresa Brasileira de Pesquisa Agropecuária, EMBRAPA, Brasil. E-mail: <juliano@cnpsa.embrapa.br>.
} 


\section{INTRODUÇÃO}

O uso de espécies florestais nativas para produção de madeira ou para enriquecimento de matas e recuperação de áreas degradadas, é dificultado pela carência de informações sobre suas exigências nutricionais nas fases de produção de mudas, estabelecimento e desenvolvimento no campo (GONÇALVES et al., 2012). Preliminarmente, sabe-se que espécies pioneiras e secundárias iniciais, por possuírem maiores taxas de crescimento, apresentam maior demanda por nutrientes, bem como maior capacidade de absorção e acúmulo destes nos tecidos vegetais, devendo, então, receber fertilização mais criteriosa, por vezes mais elevada do que a das demais classes (GONÇALVES et al., 2000).

As espécies pioneiras, comparadas às espécies climácicas, apresentam maior eficiência nutricional no tocante ao fósforo $(\mathrm{P})$. No entanto, com relação ao nitrogênio $(\mathrm{N})$ e cálcio $(\mathrm{Ca})$, ambas têm eficiência nutricional semelhante (GONÇALVES et al., 1992). É importante ressaltar que o $\mathrm{N}$ é o nutriente exigido em maior quantidade pelas culturas, refletindo elevado consumo mundial de fertilizantes nitrogenados, que supera em muito as quantidades utilizadas de $\mathrm{P}$ ou potássio (K) (RAIJ, 1991). Uma adequada nutrição nitrogenada melhora os teores foliares de $\mathrm{K}$ e de outros elementos, especialmente $\mathrm{P}$, aumentando, consequentemente, $\mathrm{o}$ crescimento e a produção (BONNEAU et al., 1993).

Uma das espécies nativas de grande importância econômica para o Brasil, devido à sua ampla utilização em marcenarias, construção naval e aeronáutica, é Cedrela fissilis Vell. (família Meliaceae), denominada popularmente cedro (XAVIER et al., 2003). Este pertence ao grupo das espécies sucessionais secundárias iniciais a secundárias tardias e ocorre desde o Estado do Pará até o Rio Grande do Sul. Além do seu valor madeireiro, trata-se de espécie melífera que pode ser empregada em projetos de arborização urbana (LORENZI, 2000), bem como é especialmente recomendada na recuperação de áreas degradadas e de matas ciliares não inundáveis (DURIGAN et al., 2002).

Em razão de sua importância econômica e ecológica e por estar no grupo das espécies ameaçadas de extinção no ecossistema brasileiro (PAULILO et al., 2007), alguns estudos relacionados à nutrição do cedro foram realizados visando à melhoria da qualidade das mudas. Entre esses estudos, pode-se citar o de Silva e Muniz (1995), que, estudando o desenvolvimento de mudas de cedro em solução nutritiva pela omissão de nutrientes, constataram que o $\mathrm{N}$ foi o nutriente que mais limitou o crescimento das mudas. Para Souza et al. (2009), que realizaram estudo com omissão de nutrientes em Latossolo Vermelho, os nutrientes $\mathrm{N}$ e $\mathrm{P}$ devem ser priorizados sem estudos envolvendo adubação do cedro.

Em relação à adubação nitrogenada, Renó et al. (1997) verificaram que mudas de cedro cultivadas por 170 dias em Latossolo Vermelho-Amarelo apresentaram alturas médias de 14,4 e 8,6 cm, respectivamente, para tratamento completo com macro e micronutrientes e tratamento com omissão de N. Esses autores constataram ainda que a omissão de $\mathrm{N}$ resultou em menores teores de $\mathrm{P}, \mathrm{K}, \mathrm{Mg}, \mathrm{Mn}, \mathrm{Cu}$ e $\mathrm{Zn}$ na parte aérea de cedro.

No âmbito da adubação nitrogenada para mudas de cedro, verificou-se que os trabalhos da literatura, seja em solução nutritiva, seja em solo, utilizaram a técnica do elemento faltante e as mudas foram desenvolvidas em períodos de até 170 dias. Assim, em razão da necessidade de estudar e indicar doses adequadas de $\mathrm{N}$ para o crescimento inicial do cedro, bem como a influência do $\mathrm{N}$ no metabolismo de absorção de nutrientes, objetivou-se avaliar o efeito de doses de $\mathrm{N}$ no crescimento inicial, teor e acúmulo de nutrientes em mudas da espécie cultivadas por 210 dias.

\section{MATERIAL E MÉTODOS}

O experimento foi realizado entre os meses de novembro de 2007 e junho de 2008 em estufa plástica (sem controle de temperatura e umidade relativa do ar), no Departamento de Recursos Naturais da FCA/UNESP, em Botucatu, SP, cujas coordenadas geográficas são $22^{\circ} 40^{\prime} 31^{\prime \prime} \mathrm{S}$ e $48^{\circ} 25^{\prime} 37^{\prime \prime} \mathrm{W}$ e a altitude média é de $770 \mathrm{~m}$. O delineamento experimental utilizado foi de blocos casualizados com cinco tratamentos, que testavam doses de $\mathrm{N}$ e quatro repetições. As doses testadas foram de $0,40,80,120$ e $160 \mathrm{mg} \mathrm{dm}^{-3} \mathrm{~N}$.

Utilizou-se um Latossolo Vermelho de textura média (EMBRAPA, 2006), coletado da camada subsuperficial em área experimental da FCA/UNESP e peneirado em malha de $2 \mathrm{~mm}$. Uma amostra desse solo foi coletada para análises química e granulométrica, conforme metodologias descritas por Raij et al. (2001) e Embrapa (1997), respectivamente. De acordo com os resultados das análises, o solo apresentava 289, 56 e $655 \mathrm{~g} \mathrm{dm}^{-3} \mathrm{de}$ argila, silte e areia, respectivamente; valor de $\mathrm{pH}$ $\left(\mathrm{CaCl}_{2} 1 \mathrm{~mol} \mathrm{~L}^{-1}\right)$ de 4,1; $26 \mathrm{~g} \mathrm{dm}^{-3}$ de M.O.; 4,0 $\mathrm{mg} \mathrm{dm}^{-3} \mathrm{de}$ 
$\mathrm{P}_{\text {resina }} ; 0,1 \mathrm{mmol}_{\mathrm{c}} \mathrm{dm}^{-3} \mathrm{de} \mathrm{K} ; 2,0 \mathrm{mmol}_{\mathrm{c}} \mathrm{dm}^{-3} \mathrm{deCa}^{2} 0,8 \mathrm{mmol}_{\mathrm{c}}$ $\mathrm{dm}^{-3} \mathrm{de} \mathrm{Mg} ; 2,9 \mathrm{mmol}_{\mathrm{c}} \mathrm{dm}^{-3}$ de soma de bases; $81 \mathrm{mmol}_{\mathrm{c}}$ $\mathrm{dm}^{-3}$ de CTC; e $3 \%$ de saturação por bases. Para os micronutrientes $\mathrm{B}, \mathrm{Fe}, \mathrm{Mn}, \mathrm{Cu}$ e $\mathrm{Zn}$, verificaram-se valores de 0,$21 ; 79 ; 0,6 ; 0,8$; e $0,1 \mathrm{mg} \mathrm{dm}^{-3}$, respectivamente.

A saturação por bases do solo foi corrigida para $60 \%$, através da incorporação de calcário dolomítico (29\% de $\mathrm{CaO} ; 18 \%$ de $\mathrm{MgO}$ e PRNT de $96 \%$ ). O solo foi incubado dentro de vasos plásticos de $20 \mathrm{dm}^{-3}$ por um período de 40 dias, o qual foi mantido umedecido. O cálculo de necessidade de calagem foi realizado de acordo com Raij et al. (2001), e cada vaso recebeu 48,1 g de calcário. Passado o período de incubação, incorporaram-se os fertilizantes ao solo. Na adubação com doses de N, utilizou-se como fonte a ureia, e todos os tratamentos receberam $100 \mathrm{mg} \mathrm{dm}^{-3} \mathrm{de} \mathrm{P}$ e $80 \mathrm{mg} \mathrm{dm}^{-3} \mathrm{de} \mathrm{K}$, nas formas de superfosfato triplo e cloreto de potássio.

Após a correção e adubação do solo, uma muda de cedro (Cedrela fissilis Vell.) foi plantada em cada unidade experimental, constituída de um vaso plástico preenchido com $20 \mathrm{dm}^{-3}$ de solo. Após 210 dias do plantio, as mudas foram avaliadas em altura (do nível do solo até a gema apical utilizando régua graduada) e diâmetro de colo (medido a $2 \mathrm{~cm}$ da superfície com paquímetro digital), e determinou-se a média de índice SPAD das folhas de quatro ramos cardeais medianos ao caule (utilizando-se e um medidor portátil de clorofila marca Minolta ${ }^{\circledR}$, modelo SPAD-502). Todas as folhas foram destacadas para determinação da área foliar no medidor Area Meter (Modelo LICOR LI-3000). Cada muda foi dividida em folhas, caule e raiz, e cada parte da planta foi lavada em água deionizada e acondicionada em saco de papel, permanecendo em estufa de secagem a $60{ }^{\circ} \mathrm{C}$, com circulação de ar até massa constante.

Realizada a pesagem da massa seca das partes da planta, estas foram moídas em moinho tipo Willey e amostradas para quantificação dos teores de macro (N, P, K, Ca, Mg e S) e micronutrientes (B, Cu, Fe, Mn e Zn), conforme metodologias descritas em Malavolta et al. (1997). A quantidade de cada nutriente absorvida pela planta foi obtida pelo somatório das quantidades acumuladas nas folhas, caule e raiz. O acúmulo de nutrientes em cada uma dessas partes foi calculado pela multiplicação da respectiva massa seca pela concentração de cada nutriente e posterior divisão por 1.000, obtendo-se o resultado em g e mg para macro e micronutrientes, respectivamente.
Os resultados foram submetidos à análise de variância através do programa SISVAR (FERREIRA, 2004), e, quando verificado o efeito significativo das doses, estas foram analisadas por regressão, ajustandose as equações de acordo com a significância e valor de F.

\section{RESULTADOS}

As equações de regressão para altura, diâmetro de colo, área foliar, índice SPAD, massas secas de folha, de caule, de raiz e massa seca total ajustaram-se a um modelo linear crescente (Tabela 1), e as médias verificadas com a máxima dose de $\mathrm{N}$ corresponderam, respectivamente, a 11,46 cm; $1,8 \mathrm{~cm} ; 1.242,92 \mathrm{~cm}^{2} ; 27,83$; $10,39 \mathrm{~g} ; 13,31 \mathrm{~g} ; 38,82 \mathrm{~g}$; e $63,54 \mathrm{~g}$.

Os teores de macronutrientes nas folhas, correspondentes a 11,$35 ; 1,21 ; 10,0 ; 18,85 ; 2,62$; e $3,35 \mathrm{~g} \mathrm{~kg}^{-1}$ para $\mathrm{N}, \mathrm{P}, \mathrm{K}, \mathrm{Ca}, \mathrm{Mg}$ e S, respectivamente (Tabela 2), não foram afetados pelas doses de N. Com relação aos teores foliares de micronutrientes, constatou-se efeito dos tratamentos apenas no teor de B, cuja equação de regressão se ajustou a um modelo linear decrescente, e com a menor e maior dose de $\mathrm{N}$ o teor de $\mathrm{B}$ correspondeu, respectivamente, a 57,45 e $21,85 \mathrm{mg} \mathrm{kg}^{-1}$. Os valores 6,$35 ; 250,4 ; 20,8$; e $15,45 \mathrm{mg} \mathrm{kg}^{-1}$ corresponderam aos teores de $\mathrm{Cu}$, $\mathrm{Fe}, \mathrm{Mn}$ e Zn, respectivamente (Tabela 3 ).

No caule não houve efeito dos tratamentos para teores de macronutrientes. Os teores de N, P, K, Ca, $\mathrm{Mg}$ e S foram, respectivamente, de 6,9; 1,28; 8,25; 7,3; 1,65; e 1,32 $\mathrm{g} \mathrm{kg}^{-1}$ (Tabela 2). Para os micronutrientes, apenas o teor de $\mathrm{Cu}$ variou em função das doses de $\mathrm{N}$, ajustando-se a um modelo linear decrescente (Tabela 3). A diferença entre os teores máximo e mínimo de $\mathrm{Cu}$ foi de $1,4 \mathrm{mg} \mathrm{kg}^{-1}$, e com a dose máxima de $\mathrm{N}$ o teor deste micronutriente foi de $4,19 \mathrm{mg} \mathrm{kg}^{-1}$.

$\mathrm{Na}$ raiz, verificou-se efeito das doses de $\mathrm{N}$ nos teores de $\mathrm{Mg}, \mathrm{Cu}$ e Mn (Tabelas 2 e 3). A equação de regressão do teor de $\mathrm{Mg}$ ajustou-se a um modelo linear crescente e apresentou teor máximo de $2,82 \mathrm{~g} \mathrm{~kg}^{-1}$. Os teores de N, P, K, Ca e S foram de 7,8; 1,49; 9,8; 3,15; e 1,79 $\mathrm{g} \mathrm{kg}^{-1}$, respectivamente (Tabela 2). Para os teores de $\mathrm{Cu}$ e Mn, as equações de regressão ajustaram-se a um modelo linear crescente e apresentaram valores máximos de 11,74 e 46,46 $\mathrm{mg} \mathrm{kg}^{-1}$, respectivamente. Os teores de $\mathrm{B}, \mathrm{Fe}$ e $\mathrm{Zn}$ foram, respectivamente, de 23,75; 1.274,8; e 12,35 $\mathrm{mg} \mathrm{kg}^{-1}$ (Tabela 3).

Revista Árvore, Viçosa-MG, v.37, n.3, p.385-392, 2013 
Tabela 1 - Equações de regressão referentes a altura, diâmetro de colo, área foliar, índice SPAD, matéria seca de folhas, matéria seca de caule, matéria seca de raiz e matéria seca total de mudas de cedro (Cedrela fissilis Vell.), em função de doses de nitrogênio.

Table 1 -Regression equations for height, lap diameter, leaf area, SPA Dindex, leaves dry matter, stem dry matter, root dry matter and total dry matter of cedar seedlings (Cedrela fissilis Vell.) as affected by nitrogen rates.

\begin{tabular}{lcc}
\hline Parâmetro & Equação de regressão & $\mathrm{R}^{2} \mathrm{e}$ significância \\
\hline Altura & $\mathrm{y}=0,0700 \mathrm{x}+0,2596$ & $0,65^{* *}$ \\
Diâmetro & $\mathrm{y}=0,0043 \mathrm{x}+1,1565$ & $0,88^{* *}$ \\
Área foliar & $\mathrm{y}=5,3413 \mathrm{x}+388,3095$ & $0,76^{* *}$ \\
Índice SPAD & $\mathrm{y}=0,0662 \mathrm{x}+17,2385$ & $0,91 * *$ \\
Matéria seca de folha & $\mathrm{y}=0,0611 \mathrm{x}+0,6105$ & $0,94 * *$ \\
Matéria seca de caule & $\mathrm{y}=0,0513 \mathrm{x}+5,1050$ & $0,92 * *$ \\
Matéria seca de raiz & $\mathrm{y}=0,1438 \mathrm{x}+15,8115$ & $0,96 * *$ \\
Matéria seca total & $\mathrm{y}=0,2562 \mathrm{x}+22,5355$ & $0,99 * *$ \\
\hline
\end{tabular}

$* *$ significativo a $1 \%$.

Tabela 2 - Equações de regressão referentes aos teores de macronutrientes em folhas, caule e raiz de mudas de cedro (Cedrela fissilis Vell.), em função de doses de nitrogênio.

Table 2 - Regression equations for macronutrients content in leaves, stem, and root of cedar seedlings (Cedrela fissilis Vell.) as affected by nitrogen rates.

\begin{tabular}{|c|c|c|c|}
\hline Nutriente & $\begin{array}{l}\text { Parte da } \\
\text { planta }\end{array}$ & $\begin{array}{c}\text { Equação } \\
\text { de regressão }\end{array}$ & $\begin{array}{c}\mathrm{R}^{2} \mathrm{e} \\
\text { significância }\end{array}$ \\
\hline \multirow{3}{*}{$\mathrm{N}$} & Folha & ns $(y=11,35)$ & - \\
\hline & Caule & $\mathrm{ns}(\mathrm{y}=6,90)$ & - \\
\hline & Raiz & ns $(y=7,80)$ & - \\
\hline \multirow{3}{*}{$\mathrm{P}$} & Folha & ns $(y=1,21)$ & - \\
\hline & Caule & ns $(y=1,28)$ & - \\
\hline & Raiz & ns $(y=1,49)$ & - \\
\hline \multirow{3}{*}{$\mathrm{K}$} & Folha & ns $(y=10,0)$ & - \\
\hline & Caule & $\mathrm{ns}(\mathrm{y}=8,25)$ & - \\
\hline & Raiz & ns $(y=9,80)$ & - \\
\hline \multirow{3}{*}{$\mathrm{Ca}$} & Folha & ns $(y=18,85)$ & - \\
\hline & Caule & ns $(y=7,30)$ & - \\
\hline & Raiz & $\mathrm{ns}(\mathrm{y}=3,15)$ & - \\
\hline \multirow{3}{*}{$\mathrm{Mg}$} & Folha & $\mathrm{ns}(\mathrm{y}=2,62)$ & - \\
\hline & Caule & ns $(y=1,65)$ & - \\
\hline & Raiz & $y=0,0053 x+1,975$ & $0,46^{*}$ \\
\hline \multirow{3}{*}{$\mathrm{S}$} & Folha & $\mathrm{ns}(\mathrm{y}=3,35)$ & - \\
\hline & Caule & $\mathrm{ns}(\mathrm{y}=1,32)$ & - \\
\hline & Raiz & ns $(y=1,79)$ & - \\
\hline
\end{tabular}

* significativo a $5 \%$.

As equações de regressão do acúmulo total de macro e micronutrientes ajustaram-se a modelos lineares crescentes, e, com a maior dose de $\mathrm{N}$, as mudas de cedro acumularam 0,$48 ; 0,08 ; 0,37 ; 0,38 ; 0,15 ;$ e $0,46 \mathrm{~g}$ de $\mathrm{N}$, $\mathrm{P}, \mathrm{K}, \mathrm{Ca}, \mathrm{Mg}$ e S por planta, respectivamente (Tabela 4). Com a mesma dose de $\mathrm{N}$, o máximo acúmulo de $\mathrm{B}, \mathrm{Cu}$,
$\mathrm{Fe}, \mathrm{Mn}$ e $\mathrm{Zn}$ correspondeu, respectivamente, a 1,39; 0,$55 ; 65,85 ; 2,16$; e $0,75 \mathrm{mg}$ por planta (Tabela 4).

\section{DISCUSSÃO}

Os incrementos em altura, diâmetro, área foliar e massa seca das mudas de cedro em razão do aumento das doses de $\mathrm{N}$ evidenciam a importância da adubação nitrogenada no desenvolvimento inicial dessa espécie. Renó et al. (1997) também constataram que o Né altamente requerido pelo cedro no início de seu desenvolvimento, uma vez que a omissão desse nutriente acarretou reduções de $40 \%$ em altura e $57 \%$ no incremento de massa seca de parte aérea. Venturin et al. (1996) verificaram que o N é o nutriente mais importante para a produção de massa seca da parte aérea de mudas de Copaifera langsdorffii (óleo-de-copaíba). De maneira geral, essas respostas devem-se ao fato de que grandes quantidades de $\mathrm{N}$ são requeridas pelas plantas, principalmente na fase inicial de desenvolvimento (MALAVOLTA et al., 1997). A restrição de N leva à redução de crescimento, pois esse nutriente, além de fazer parte da estrutura de aminoácidos, proteínas, bases nitrogenadas, ácidos nucleicos, enzimas, vitaminas, pigmentos e produtos secundários, participa de processos como absorção iônica, fotossíntese, respiração, multiplicação e diferenciação celular (MARSCHNER, 1995).

Como já comentado, os efeitos da adubação nitrogenada em cedro foram estudados por Renó et al. (1997), que verificaram também que mudas de cedro cultivadas por 170 dias em Latossolo Vermelho-Amarelo apresentaram alturas médias de 14,4 e 8,6 cm, para tratamento completo com macro e micronutrientes e tratamento com omissão de N, respectivamente. Esses

Revista Árvore, Viçosa-MG, v.37, n.3, p.385-392, 2013 
autores obtiveram maior incremento em altura em menor tempo de cultivo, provavelmente pelo fato de a dose de $\mathrm{N}$ utilizada no plantio das mudas, somada à dose de cobertura, totalizar $229 \mathrm{mg} \mathrm{dm}^{-3} \mathrm{~N}$, ou seja, $43 \%$ maior do que a dose utilizada neste estudo.

Tabela 3 - Equações de regressão referentes aos teores de micronutrientes em folhas, caule e raiz de mudas de cedro (Cedrela fissilis Vell.), em função de doses de nitrogênio.

Table 3 -Regression equations for micronutrients content in leaves, stem, and root of cedar seedlings (Cedrela fissilis Vell.) as affected by nitrogen rates.

\begin{tabular}{|c|c|c|c|}
\hline Nutriente & $\begin{array}{l}\text { Parte da } \\
\text { planta }\end{array}$ & $\begin{array}{c}\text { Equação } \\
\text { de regressão }\end{array}$ & $\begin{array}{c}\mathrm{R}^{2} \mathrm{e} \\
\text { significância }\end{array}$ \\
\hline \multirow{3}{*}{ B } & Folha & $y=-0,2225 x+57,4500$ & $0,83 * *$ \\
\hline & Caule & $\mathrm{ns}(\mathrm{y}=11,85)$ & - \\
\hline & Raiz & ns $(y=23,75)$ & - \\
\hline \multirow{3}{*}{$\mathrm{Cu}$} & Folha & $\mathrm{ns}(\mathrm{y}=6,35)$ & - \\
\hline & Caule & $y=-0,0088 x+5,600$ & $0,92 * *$ \\
\hline & Raiz & $y=0,0412 x+5,150$ & $0,55 * *$ \\
\hline \multirow{3}{*}{$\mathrm{Fe}$} & Folha & ns $(y=250,4)$ & - \\
\hline & Caule & $\mathrm{ns}(\mathrm{y}=83,8)$ & - \\
\hline & Raiz & ns $(y=1274,8)$ & - \\
\hline \multirow{3}{*}{ Mn } & Folha & ns $(y=20,80)$ & - \\
\hline & Caule & ns $(y=14,10)$ & $0,77 * *$ \\
\hline & Raiz & $y=0,1838 x+17,050$ & - \\
\hline \multirow{3}{*}{$\mathrm{Zn}$} & Folha & ns $(y=15,45)$ & - \\
\hline & Caule & ns $(y=11,10)$ & - \\
\hline & Raiz & ns $(y=12,35)$ & $0,46^{*}$ \\
\hline
\end{tabular}

* significativo a $1 \%$.
A adubação nitrogenada promoveu incrementos de área foliar (Tabela 1), provavelmente pelo fato de o N estimular o crescimento vegetativo (MALAVOLTA et al., 1997). Em casos de baixo suprimento de N, normalmente há redução na produção e tamanho de folhas (MAFFEIS et al., 2000). Para mudas de Rhapis excelsa (palmeira-ráfia) cultivadas em Latossolo Vermelho, Luz et al. (2006) verificaram que o $\mathrm{N}$ foi o nutriente mais importante para o desenvolvimento de folhas, obtendo incremento de $13 \%$ na área foliar, em comparação com mudas que não receberam adubação com esse nutriente.

O índice SPAD é um parâmetro utilizado para indicar o teor de clorofila na folha e geralmente se correlaciona positivamente com o teor de $\mathrm{N}$ na planta, motivo pelo qual pode ser usado para estimar a quantidade de $\mathrm{N}$ absorvida do solo (SCHADCHINA; DMITRIEVA, 1995). Essa relação é atribuída pelo fato de que 50 a $70 \%$ do $\mathrm{N}$ total das folhas se integra a enzimas (CHAPMAN; BARRETO, 1997), que estão associadas aos cloroplastos (STOCKING; ONGUN, 1962). Em razão disso, trabalhos têm demonstrado correlação entre leituras índice SPAD e o "status" de N em diversas culturas (ARGENTA et al., 2001). Contudo, ao contrário do que se esperava, essa relação não foi observada neste estudo, uma vez que o teor de $\mathrm{N}$ na folha permaneceu inalterado, independentemente da dose de $\mathrm{N}$ (Tabela 2), enquanto o índice SPAD aumentou linearmente com o incremento da adubação nitrogenada (Tabela 1). Supõe-se, então, que a leitura do índice SPAD em cedro esteja mais

Tabela 4 - Equações de regressão referentes ao acúmulo total de macro e micronutrientes em mudas de cedro (Cedrela fissilis Vell.), em função de doses de nitrogênio.

Table 4 - Regression equations for total accumulation of macro and micronutrients in cedar seedlings (Cedrela fissilis Vell.) as affected by nitrogen rates.

\begin{tabular}{ccc}
\hline Parâmetro & Equação de regressão & $\mathrm{R}^{2}$ e significância \\
\hline $\mathrm{N}$ & $\mathrm{y}=0,0020 \mathrm{x}+0,1618$ & $0,99 * *$ \\
$\mathrm{P}$ & $\mathrm{y}=0,0003 \mathrm{x}+0,0333$ & $0,99 * *$ \\
$\mathrm{~K}$ & $\mathrm{y}=0,0010 \mathrm{x}+0,2169$ & $0,94 * *$ \\
$\mathrm{Ca}$ & $\mathrm{y}=0,0015 \mathrm{x}+0,1421$ & $0,95 * *$ \\
$\mathrm{Mg}$ & $\mathrm{y}=0,0007 \mathrm{x}+0,0393$ & $0,96^{*}$ \\
$\mathrm{~S}$ & $\mathrm{y}=0,0005 \mathrm{x}+0,3785$ & $0,98^{* *}$ \\
$\mathrm{~B}$ & $\mathrm{y}=0,0055 \mathrm{x}+0,5080$ & $0,97 * *$ \\
$\mathrm{Cu}$ & $\mathrm{y}=0,0029 \mathrm{x}+0,0872$ & $0,80^{* *}$ \\
$\mathrm{Fe}$ & $\mathrm{y}=0,4062 \mathrm{x}+0,8566$ & $0,92^{* *}$ \\
$\mathrm{Mn}$ & $\mathrm{y}=0,0118 \mathrm{x}+0,2759$ & $0,95 * *$ \\
$\mathrm{Zn}$ & $\mathrm{y}=0,0032 \mathrm{x}+0,2372$ & $0,95 * *$ \\
\hline
\end{tabular}

** significativo a $1 \%$ e $*$ significativo a $5 \%$. 
associada ao teor de clorofila do que ao teor de N. Contudo, essa hipótese não pode ser comprovada com esse único experimento. Para reforçar esse resultado ou, mesmo, averiguar os fatores que possam influenciar essa resposta, são necessários estudos mais apurados que viabilizem o manejo da nutrição nitrogenada no cedro com base na leitura SPAD.

De modo geral, doses baixas de $\mathrm{N}$ limitaram o crescimento das plantas de cedro, mas não a absorção de nutrientes. Tal afirmação é embasada no fato de que os teores da maioria dos nutrientes na raiz, no caule e nas folhas mantiveram-se semelhantes, independentemente da dose de N (Tabelas 2 e 3). Visando ao manejo de nutrição do cedro, os teores de nutrientes das folhas são os mais importantes. Na folha, o único nutriente afetado foi o $\mathrm{B}$, que teve seu teor reduzido com o aumento da dose de N (Tabela 3). Efeito semelhante foi verificado por Souza et al. (2009), que obtiveram maiores teores foliares de $\mathrm{B}$ em plantas de cedro cultivadas em Latossolo Vermelho-Amarelo com omissão de adubação nitrogenada. Esses resultados possivelmente ocorreram em razão do efeito antagônico entre a absorção de $\mathrm{N}$ e $\mathrm{B}$, que pode ocorrer em qualquer espécie vegetal (MALAVOLTA et al., 1997). Entretanto, segundo esses mesmos autores, mesmo com a maior dose de $\mathrm{N}$ o teor foliar de $\mathrm{B}\left(21,8 \mathrm{mg} \mathrm{kg}^{-1}\right)$ permaneceu acima do considerado crítico para esse nutriente na folha, que é de $15 \mathrm{mg} \mathrm{kg}^{-1}$.

Os teores de $\mathrm{Cu}$ na raiz e no caule apresentaram comportamentos desiguais com a variação da dose de $\mathrm{N}$, indicando que a adubação nitrogenada afeta a translocação desse nutriente em plantas de cedro. O aumento da dose de $\mathrm{N}$ reduziu o teor de $\mathrm{Cu}$ no caule, ao contrário do ocorrido na raiz (Tabela 3 ). Isso acontece porque o $\mathrm{N}$ pode afetar a disponibilidade e mobilidade do $\mathrm{Cu}$ nas plantas, podendo o seu excesso causar deficiência deste nutriente em função de interações não específicas (MATTOS JÚNIOR et al., 2010). Já com relação ao Fe, embora o seu teor não tenha sido afetado pelas doses de N, verificou-se que o seu teor na raiz foi 5,1 e 15,2 vezes superior ao encontrado nas folhas e no caule, respectivamente (Tabela 3). Distribuição desigual de $\mathrm{Fe}$ entre a raiz e a parte aérea também foi verificada por Barroso et al. (2005) em Tectona grandis (teca), por Missio e Nicoloso (2005) em Apuleia leiocarpa (grápia) e por Silveira et al. (2003) em Eucalyptus grandis (eucalipto). Em relação ao metabolismo do Fe na planta, deve-se levar em conta que esse elemento apresenta baixa mobilidade nos tecidos vegetais, e essa mobilidade

Revista Árvore, Viçosa-MG, v.37, n.3, p.385-392, 2013 é negativamente afetada por alguns fatores, como elevado teor de Mn (DECHEN; NACHTIGALL, 2006). De fato, o que se observou neste estudo foi o aumento linear do teor de Mn na raiz, o que poderia justificar a baixa mobilidade do Fe para a parte aérea.

O aumento de acúmulo de nutrientes nas plantas de cedro em função do aumento das doses de N (Tabela 5) se deveu mais ao incremento de produção de matéria seca do que a variações nos teores de nutrientes. Contudo, observou-se alteração na dinâmica de absorção dos nutrientes pelas mudas com a variação da adubação nitrogenada, o que evidencia a influência de doses de $\mathrm{N}$ na dinâmica de absorção de nutrientes por mudas de cedro. Assim, com a dose de $160 \mathrm{mg} \mathrm{dm}^{-3} \mathrm{~N}$, a ordem de absorção de nutrientes pelas plantas de cedro foi a seguinte: $\mathrm{N}>\mathrm{S}>\mathrm{Ca}>\mathrm{K}>\mathrm{Mg}>\mathrm{P}>\mathrm{Fe}>\mathrm{Mn}>\mathrm{B}>\mathrm{Zn}>$ $\mathrm{Cu}$. Já com a dose $0 \mathrm{mg} \mathrm{dm}^{-3}$ de $\mathrm{N}$ a absorção de nutrientes seguiu esta ordem: $\mathrm{S}>\mathrm{K}>\mathrm{N}>\mathrm{Ca}>\mathrm{Mg}>\mathrm{P}>\mathrm{Fe}>\mathrm{B}>\mathrm{Mn}>$ $\mathrm{Zn}>\mathrm{Cu}$.

\section{CONCLUSÕES}

De modo geral, as menores doses de $\mathrm{N}$ limitaram o crescimento, mas não a absorção de nutrientes pelas plantas de cedro.

Na folha, o B foi o único nutriente afetado pela adubação nitrogenada e teve seu teor reduzido com o aumento das doses de N.

O aumento da adubação nitrogenada alterou a distribuição do $\mathrm{Cu}$ nas plantas, promovendo aumento no teor desse nutriente na raiz e redução de seu teor no caule.

Maiores doses de $\mathrm{N}$ promoveram maior acúmulo de todos os nutrientes em função do incremento de produção de matéria seca.

A adubação nitrogenada modificou a dinâmica de absorção dos nutrientes em cedro, e, com a dose de $160 \mathrm{mg} \mathrm{dm}^{-3} \mathrm{~N}$, a quantidade absorvida obedeceu à seguinte ordem: $\mathrm{N}>\mathrm{S}>\mathrm{Ca}>\mathrm{K}>\mathrm{Mg}>\mathrm{P}>\mathrm{Fe}>\mathrm{Mn}>\mathrm{B}>$ $\mathrm{Zn}>\mathrm{Cu}$.

\section{REFERÊNCIAS}

ARGENTA, G.; SILVA, P. R. F.; BORTOLINI, C. G. Clorofila na folha como indicador do nível de nitrogênio em cereais. Ciência Rural, v.31, n.4, p.715-722, 2001. 
BARROSO, D. G. et al. Diagnóstico de deficiência de macronutrientes em mudas de teca. Revista Árvore, v.29, n.5, p.671-679, 2005.

BONNEAU, X. et al. Nutrition minérale des cocotiers hybrides sur tourbe de la pépinière à l'entrée en production. Oléagineux, v.48, p.9-26, 1993.

CHAPMAN, S. C.; BARRETO, H. J. Using a chlorophyll meter to estimate specific leaf nitrogen of tropical maize during vegetative growth. Agronomy Journal, v.89, n.4, p.557-562, 1997.

DECHEN, A. R.; NATCHTIGALL, G.R. Micronutrientes. In: FERNANDES, M.S. (Ed.). Nutrição mineral de plantas. Viçosa, MG,: SBCS, 2006. p.328-354.

DURIGAN, G. et al. Sementes e mudas de árvores tropicais. 2.ed. São Paulo: Páginas \& Letras Editora e Gráfica, 2002. 65p.

\section{EMPRESA BRASILEIRA DE PESQUISA} AGROPECUÁRIA - EMBRAPA. Centro Nacional de Pesquisa de Solos - CNPS. Sistema Brasileiro de classificação de solos. Brasília: 2006. 412p.

\section{EMPRESA BRASILEIRA DE PESQUISA} AGROPECUÁRIA - EMBRAPA. Centro Nacional de Pesquisa de Solos - CNPS. Manual de métodos de análises de solos. 2 ed. Rio de Janeiro: Serviço Nacional de Levantamento e Conservação de Solos, 1997. 212p.

FERREIRA, D. F. Sisvar versão 4.6. Lavras: DEX/UFLA, 2004. 32p.

GONÇALVES, E. O. et al. Nutrição de mudas de angico-vermelho (Anadenanthera macrocarpa (Benth.) Brenan) submetidas a doses de N, P, K, Ca E Mg. Revista Árvore, v.36, n.2, p. 219-228, 2012.

GONÇALVES, J. L. M. et al. Capacidade de absorção e eficiência nutricional de algumas espécies arbóreas tropicais. Revista do Instituto Florestal, v.4, Parte 2, p.463-469, 1992. Edição Especial.

GONÇALVES, J. L. M.; SANTARELLI, E. G.; MORAES NETO, S. P. Produção de mudas de espécies nativas: substrato, nutrição, sombreamento e fertilização. In: GONÇALVES, J. L. M.; BENEDETI, V. (Ed.). Nutrição e fertilização florestal. Piracicaba: IPEF, 2000. p.309-350.
LORENZI, H. Árvores brasileiras: manual de identificação e cultivo de plantas arbóreas nativas do Brasil. 3.ed. Nova Odessa: Instituto Plantarum, 2000. 368p.

LUZ, P. B. et al. Efeitos de nitrogênio, fósforo e potássio no crescimento de Rhapis excelsa (Thunberg) Henry ex. Rehder (palmeira-ráfia). Ciência e Agrotecnologia, v.30, n.3, p.429-434, 2006.

MAFFEIS, A. R.; SILVEIRA, R. L. V. A.; BRITO, J. O. Reflexos das deficiências de macronutrientes e boro no crescimento das plantas, produção e qualidade de óleo essencial em Eucalyptus citriodora. Scientia Forestalis, n.57, p.87-98, 2000.

MALAVOLTA, E.; VITTI, G. C.; OLIVEIRA, S. A. Avaliação do estado nutricional das plantas, princípios e aplicações. 2.ed. Piracicaba: POTAFOS, 1997.319p.

MARSCHNER, H. Mineral nutrition of higler plants. 2.ed. New York: Academic Press, 1995. 889p.

MATTOS JÚNIOR, D. et al. Nitrogênio e cobre na produção de mudas de citros em diferentes portaenxertos. Bragantia, v.69, n.1, p.135-147, 2010.

MISSIO, E. L.; NICOSOLO, F.T. Distúrbios nutricionais induzidos pela adubação de fósforo e ferro em plantas jovens de grápia (Apuleia leiocarpa). Ciência Florestal, v.15, n.4, p.377-389, 2005.

PAULILO, M. T. S. et. al. Responses to light changes in tropical seedlings of the Brazilian Atlantic rainforest tree species Cecropia glazioui (Cecropiaceae) and Cedrela fissilis (Meliaceae). Australian Journal of Botany, v.55, p.795-802, 2007.

RAIJ, B.van. et al. Análise química para avaliação da fertilidade de solos tropicais. Campinas: Instituto Agronômico de Campinas, 2001. 285p.

RAIJ, B. van. Fertilidade do solo e adubação. Piracicaba: Ceres/POTAFOS, 1991. 343p.

Revista Árvore, Viçosa-MG, v.37, n.3, p.385-392, 2013 
RENÓ, N. B. et al. Limitações nutricionais ao crescimento inicial de quatro espécies arbóreas nativas em Latossolo Vermelho-Amarelo. Pesquisa Agropecuária Brasileira, v.32, n.1, p.17-25, 1997.

SCHADCHINA, T. M.; DMITRIEVA, V. V. Leaf chlorophyll content as a possible diagnostic mean for the evaluation of plant nitrogen uptake from the soil. Journal of Plant Nutrition, v.18, p.1427-1437, 1995.

SILVA, M. A. G.; MUNIZ, A. S. Exigências nutricionais de mudas de cedro (Cedrela fissilis Velloso) em solução nutritiva. Revista Árvore, v.19, n.3, p.415-425, 1995.

SILVEIRA, R. L. V. A. et al. Matéria seca, concentração e acúmulo de nutrientes em mudas de Eucalyptus grandis em função da idade. Scientia Forestalis, n.64, p.136-149, 2003.
SOUZA, P. A. et al. Nutritional assessment of cedar seedlings (Cedrela fissilis; Vell.) grown in a greenhouse. Cerne, v.15, n.2, p.236-243, 2009.

STOKING, C. R.; ONGUN, A. The intracellular distribuition of some metallic elements in leaves. American Journal of Botany, v.49, n.3, p.284-289, 1962.

VENTURIN, N. et al. Fertilização de plântulas de Copaifera langsdorffii Desf. (Óleo copaíba).

Cerne, v.2, n.2, p.31-47, 1996.

XAVIER, A.; SANTOS, G. A.; OLIVEIRA, M. L. Enraizamento de miniestaca caulinar e foliar na propagação vegetativa de cedro-rosa (Cedrela fissilis Vell.). Revista Árvore, v.27, n.3, 2003. 